\title{
Triple-high expression of phosphatase and tensin homolog (PTEN), estrogen receptor (ER) and progesterone receptor (PR) may predict favorable prognosis for patients with Type I endometrial carcinoma
}

\author{
Yanfang Liang1 ${ }^{\bowtie}$, Bihua Lin², Ziyu Ye², Shasha Chen², Haibo Yu², Can Chen¹, Xin Zhang 3 , Keyuan Zhou ${ }^{2}$, \\ Jincheng Zeng ${ }^{2}$ \\ 1. Department of Pathology, Dongguan Hospital Affiliated to Medical College of Jinan University, The Fifth People's Hospital of Dongguan, Dongguan \\ 523905, China \\ 2. Dongguan Key Laboratory of Medical Bioactive Molecular Developmental and Translational Research, Guangdong Provincial Key Laboratory of Medical \\ Molecular Diagnostics, Guangdong Medical University, Dongguan, Guangdong 523808, China \\ 3. Clinical Experimental Center, Jiangmen Central Hospital, Affiliated Jiangmen Hospital of Sun Yat-sen University, Jiangmen 529030, China.
}

$\square$ Corresponding authors: Yanfang Liang, e-mail: lyfine84@126.com; tel.:+86 0769 85010001; Department of Pathology, Dongguan Hospital Affiliated to Medical College of Jinan University, The Fifth People's Hospital of Dongguan, Dongguan 523905, China. Jincheng Zeng, e-mail:zengjc@gdmu.edu.cn; tel.:+86 0769 22896440; Dongguan Key Laboratory of Medical Bioactive Molecular Developmental and Translational Research, Guangdong Medical University, Dongguan, Guangdong 523808, China.

(c) The author(s). This is an open access article distributed under the terms of the Creative Commons Attribution License (https://creativecommons.org/licenses/by/4.0/). See http://ivyspring.com/terms for full terms and conditions.

Received: 2019.01.31; Accepted: 2019.11.19; Published: 2020.01.13

\begin{abstract}
Endometrial carcinoma (EC) is the most common malignant tumors in female derived from the endometrial epithelium. Several previous studies have described estrogen receptors (ER), progesterone Receptor (PR) and phosphatase and tensin homolog (PTEN) are associated with clinicopathological factors and prognosis in EC patients. However, during EC patients follow-up, we found that some EC patients with down-regulation of PTEN, but up-regulation of ER or PR , and some EC patients with down-regulation of ER or PR, but up-regulation of PTEN also had a poor prognosis. Therefore, to reveal the prognosis of EC patients with different phenotypes based on PTEN, ER and PR expression, 120 cases formalin-fixed paraffin-embedded EC tissues and 543 cases uterine corpus endometrial carcinoma (UCEC) patients from the cancer genome atlas (TCGA) UCEC datasets were analyzed. Results showed that EC tissues can be classified to PTEN'ER'PR', PTEN ${ }^{H} E R^{L} P R^{L}$, PTEN ${ }^{H} E R^{H} P R^{H}$, PTEN ${ }^{L} E R^{H} P R^{H}$, PTEN ${ }^{H} E R^{H} P R^{L}$, PTEN ${ }^{H} E R^{L} P R^{H}$, and PTEN ${ }^{L} E R^{H} P R^{L}$ phenotypes basing on IHC analysis. Additionally, EC patients with $P T E N^{L} E R^{L} P R^{L}$ showed high malignancy, while patients with PTEN ${ }^{H} E R^{H} P R^{H}$ showed low malignancy. Therefore, combined detection of PTEN, ER, PR may help identify a small subset of EC with more aggressive behavior and may aid in risk stratification.
\end{abstract}

Key words: Endometrial carcinoma, estrogen receptors, progesterone receptor, phosphatase and tensin homolog, prognosis

\section{Introduction}

Endometrial carcinoma (EC) is the most common malignant tumors in female derived from the endometrial epithelium. Recently, the EC incidence is increased, while the 5-year survival rate is decreased
[1]. The etiology of EC is not yet clear. Experimental and epidemiologic evidence showed that the risk factors of EC including $\mathrm{BMI} \geq 25 \mathrm{~kg} / \mathrm{m}^{2}$, hypertension, diabetes, smoking, taking tamoxifen, family history of 
cancer and non-pregnant women[2, 3]. EC can be broadly classified into two types. Majority ( $80 \%)$ of EC patients are of Type I endometrioid histology, up to $15 \%$ are Type II EC patients, primarily serous carcinomas [3-5]. Most of Type I EC patients present with low-grade and early-stage disease and have a favorable prognosis. According to the previous study, $74-91 \%$ of International Federation of Gynecology and Obstetrics (FIGO) stages I-II type I EC patients have a 5-year OS time [6]. However, 10$20 \%$ of early stages I-II and $50-70 \%$ of advanced stage III-IV type I EC patients will recur after primary treatment [7]. Previous studies have revealed that clinicopathological parameters such as histological grade, FIGO clinical stage, myometrial tumor invasion, tumor size, lymph node metastasis, lymphovascular space invasion, and the patients' age and race has prognostic effect in type I EC patients[6, 8 , 9]. However, these factors are usually obtained postoperatively and have proven to be insufficient to predict recurrence and estimate survival time. Therefore, it is necessary to identify more effective prognostic predictors to identify preoperative high-risk type I EC patients.

Several previous studies have described estrogen receptors (ER), progesterone Receptor (PR) and phosphatase and tensin homolog (PTEN) are associated with clinicopathological factors and prognosis in EC patients [10-16]. Both of ER and PR are members of the nuclear receptor family that release relevant related ligand-activated transcription factors to regulate cell gene expression through activation and suppression of transcription [17-19]. Low expression or deletion of ER and PR associated with increased malignancy, invasion, and non-hormone-dependent tumor transformation [20]. The absence of PR and ER indicates poor prognosis, poor histologic type, higher histological grade, higher frequency of lymph node metastasis, and higher clinical staging at diagnosis $[18,19]$. PTEN located on chromosome 10q23.3, is known as a vital tumor suppressor gene. Recently, researchers found that PTEN has low expression during the development and progression of EC, and implies poor prognosis, higher histological grade and clinical staging, and shorter survival [21, 22]. Especially, the mutation rate of PTEN in EC is about $34 \% \sim 55 \%$, which is higher than the mutation rate of $K$-ras and P53 [23, 24]. However, during follow-up of EC patients, we found that some EC patients with down-regulation of PTEN, but up-regulation of ER or PR, and some EC patients with down-regulation of ER or PR, but up-regulation of PTEN also had a poor prognosis. Therefore, to reveal the prognosis of EC patients with different phenotypes based on PTEN, ER and PR expression,
120 cases formalin-fixed paraffin-embedded EC tissues and 543 cases UCEC patients from TCGA-UCEC datasets were analyzed.

\section{Material and Methods}

\section{Patients}

A total of 120 formalin-fixed paraffin embedded EC tissue samples between March 2005 and April 2015 from Affiliated Hospital of Guangdong Medical University (China), Dongguan Fifth People's Hospital (China), and Affiliated Jiangmen Hospital of Sun Yat-sen University (China) were collected. Tumors were staged according to the FIGO 2009 system [25]. Histological grade was assessed based on the 2014 World Health Organization criteria [26]. Patients, who had history of other tumors, underwent chemotherapy, radiotherapy, radical surgery treatment, or other anticancer therapies prior to surgery were excluded in this study. The demographic and clinical characteristics for all the patients are shown in Table 1. Informed consent was obtained from all study subjects, and the studies were approved by the institutional ethics committee.

\section{Immunohistochemistry (IHC)}

Tissue sections $(4-\mu \mathrm{M})$ were prepared from formalin-fixed paraffin embedded tissue blocks and then subjected to stain with hematoxylin and eosin as our previously described [27-29]. The results were assessed by two pathologists to demonstrate the presence of tumor and the proportion of tumor cells in each section. Tissue sections were subjected to incubate in $0.3 \%$ Hydrogen peroxide solution for 10 min at room temperature to block the endogenous peroxidase activity, and then washed by Phosphate Buffered Saline (PBS) solution. Antigenic epitopes were next retrieved by heating for $2 \mathrm{~min}$ in 10 $\mathrm{mmol} / \mathrm{L}$ citrate buffer ( $\mathrm{pH}$ 6.0). The slides were then first incubated with antibodies against PTEN (ZSGB-BIO, China), ER (ZSGB-BIO, China), PR (ZSGB-BIO, China), Ki-67 (Thermofisher,USA), p53 (Thermofisher,USA), CEA (Thermofisher,USA) and CA125 (Thermofisher,USA) for $30 \mathrm{~min}$ at room temperature. Next, the sections were washed with PBS for three times and followed by a goat anti-rabbit and mouse IgG-HRP (Kit-0015, Maixin Biotech, Fuzhou, China) secondary antibody for one hour at room temperature at 1:500 dilutions. The slides were visualized using DAB Detection Kit (Enhanced Polymer) (Kit-0015, Maixin Biotech, Fuzhou, China) and chromogenic reaction was controlled under a microscope (Nikon). After immunostaining, sections were immersed into hematoxylin for nuclear staining, then dehydrated through gradient concentrations of 
ethanol, cleared with xylene, and covered with neutral balsam.

\section{Score of immunohistochemical sections}

The score of immunohistochemical sections were assessed by two pathologists in a blinded fashion to the clinical status of the patients, as our previously reported [27-29]. The immunoreactive area (percentage of positive staining cells) and intensity scores of PTEN, ER and PR were evaluated. In brief, according to the immunoreactive area, $0-5 \%$ scored 0 , $6-25 \%$ scored $1,26-50 \%$ scored $2,51 \sim 75 \%$ scored 3 and more than $75 \%$ scored 4 . If the scores was $0 \sim 2$, the section was defined as low expression, and if the final scores was 3 6 was defined as high expression. Two specialists who were blinded to the clinical status of the patients evaluated the staining independently.

\section{mRNA expression}

All available mRNA expression data were collected from 543 UCEC tumors and 23 adjacent non-EC tissues in TCGA (https://cancergenome.nih .gov/). According to the average value of mRNA expression of each gene, EC patients were divided into 8 phenotypes $\left(P T E N^{\mathrm{L}} E S R 1^{\mathrm{L}} P G R^{\mathrm{L}}, P T E N^{\mathrm{L}} E S R 1^{\mathrm{H}}\right.$ $P G R^{\mathrm{H}}, \quad P T E N^{\mathrm{H}} E S R 1^{\mathrm{L}} P G R^{\mathrm{L}}, \quad P T E N^{\mathrm{H}} E S R 1^{\mathrm{H}} P G R^{\mathrm{H}}$, PTENLESR1LPGR ${ }^{\mathrm{H}}, P T E N^{\mathrm{L}} E S R 1^{\mathrm{H}} P G R^{\mathrm{L}}, P T E N^{\mathrm{H}} E S R 1^{\mathrm{H}}$ $P G R^{\mathrm{L}}$, and $P T E N^{\mathrm{H}} E S R 1^{\mathrm{L}} P G R^{\mathrm{H}}$ ), according to high and low PTEN, PGR and ESR1 mRNA expression.

\section{Statistical analysis}

Statistical analysis was performed using SPSS 19.0 Software (SPSS, Chicago, IL, USA). Chi-square test or Fisher's exact test was employed for analysis the differences of categorical variables. For survival analysis, overall survival (OS) or disease-free survival (DFS) was calculated using Kaplan-Meier method and evaluated by log-rank test, as our previously reported [30,31]. Multivariate analysis was based on the Cox proportional hazard regression model. A p value $<0.05$ was considered with statistical significance.

\section{Results}

\section{Classification of EC tissues based on PTEN, ER and PR expression}

It has been reported that the tumor suppressor gene PTEN is down-regulated in a variety of cancers, including breast cancer [32], prostate cancer [32] and EC [33], etc. PTEN deficiency accelerates tumuor progression and invasiveness [34], promotes macrophage infiltration [35], and plays a significant role in the pathogenesis of carcinogenesis [36]. Herein, we first analyzed the cancer genome atlas uterine corpus endometrial carcinoma (TCGA-UCEC) datasets and found that PTEN mRNA expression was down-regulated in EC tumor tissues compared with adjacent normal tissues (ANT) (Fig. 1A). Prognostic factors of EC include the presence of ER and PR. We also found that the mRNA expression of PGR encoding PR, but not ESR1 encoding ER, down-regulated in EC tissues compared with ANT in TCGA-UCEC datasets (Fig. 1A). Furthermore, correlation analysis showed that there was a significant correlation among PTEN, PGR and ESR1 mRNA expression (Fig. 1B), and they all associated with the prognosis of EC (Fig. 1C). This was also consistent with the results reported in most previous studies [18, 19, 21, 22]. To further reveal the relationship between differential expression of PTEN, ER and PR, and EC prognosis, EC patients were divided into 8 phenotypes (PTEN ${ }^{\mathrm{L} E S R} 1^{\mathrm{L}} P G R^{\mathrm{L}}$, PTENLESR1 ${ }^{\mathrm{H}} P G R^{\mathrm{H}}, P T E N^{\mathrm{H}} E S R 1^{\mathrm{L}} P G R^{\mathrm{L}}, P T E N^{\mathrm{H}} E S R 1^{\mathrm{H}}$ $P G R^{\mathrm{H}}, \quad P T E N^{L} E S R 1^{\mathrm{L}} P G R^{\mathrm{H}}, \quad P T E N^{\mathrm{L}} E S R 1^{\mathrm{H}} P G R^{\mathrm{L}}$, $P T E N^{\mathrm{H}} E S R 1^{\mathrm{H}} P G R^{\mathrm{L}}$, and PTEN $\left.{ }^{\mathrm{H}} E S R 1^{\mathrm{L}} P G R^{\mathrm{H}}\right)$, according to high $(\mathrm{H})$ and low (L) PTEN, PGR and ESR1 mRNA expression (Fig. 1D). Additionally, we collected 120 formalin-fixed paraffin-embedded EC tissues and examined PTEN, ER and PR expression by IHC analysis (Fig. E). Based on PTEN, ER and PR expression, EC tissues can be classified to PTENLERLPR ${ }^{L} \quad(48 / 120), \quad$ PTEN ${ }^{H}$ ER $^{L}{ }^{2}{ }^{L} \quad(30 / 120)$, PTEN $^{H}{ }^{H}{ }^{H}{ }^{H}{ }^{H}$ (20/120), PTENLER ${ }^{H}{ }^{H}{ }^{H} \quad(12 / 120)$, PTEN $^{H}$ ER $^{\text {HPR }}{ }^{L}$ (4/120), PTEN ${ }^{H}$ ER $^{L}{ }^{2}{ }^{\mathrm{H}}(4 / 120)$, and PTENLER $^{H P R}{ }^{L}$ (2/120) phenotype (Fig. 1F). The demographic and clinical characteristics for all EC phenotypes are shown in Table $1.60 \%$ of EC patients with PTEN ${ }^{H} E R^{L}{ }^{L} R^{L}$ and PTEN ${ }^{H} E R^{H}{ }^{H} R^{H}$ phenotype were G1 histological grading, respectively, while $20.83 \%$ of EC patients with PTENLERLPR ${ }^{L}$ phenotype were G1 histological grading (Table 1). Similarly, in FIGO clinical staging, most EC patients with PTEN $^{\mathrm{H} E R}{ }^{\mathrm{L} P R}{ }^{\mathrm{L}}(66.7 \%)$ and PTEN ${ }^{\mathrm{H}} \mathrm{ER}^{\mathrm{H} P R^{\mathrm{H}}}(45.0 \%)$ phenotype were stage I, while $25.00 \%$ of EC patients with PTENLERLPRL phenotype were stage I (Table 1). These results suggest that different EC phenotypes which classified by PTEN, ER and PR expression may be associated with clinical pathological and histological grading.

\section{EC patients with triple-high expression of PTEN, ER and PR showed a lower degree of malignancy and proliferative activity}

To reveal the proliferative activity of EC patients with different phenotypes, Ki-67 and p53 were detected by IHC analysis (Fig. 2A). Results showed that Ki-67 was low expressed in EC patients with PTEN ${ }^{H} E R^{L}{ }^{2}{ }^{L}$ and PTENHER ${ }^{H} R^{H}$ phenotype, while high expressed in EC patients with PTENLERLPR ${ }^{L}$ phenotype (Fig. 2B). Indeed, based on TCGA-UCEC 
datasets, we also found that EC patients with $P T E N^{L} E S R 1^{L} P G R^{L}$ phenotype had high expression of Ki67 mRNA, while patients with PTEN ${ }^{\mathrm{H}} E S R 1^{\mathrm{H}} P G R^{\mathrm{H}}$ phenotype had low expression of Ki67 mRNA (Fig. 2C). Simultaneously, we also found p53 was low expressed in EC patients with PTEN ${ }^{H} E^{H} \mathrm{PR}^{\mathrm{H}}$ phenotype, and TP53 (encoding p53) mRNA was low expressed in EC patients with PTEN ${ }^{\mathrm{H}} E S R 1^{\mathrm{H} P G R} \mathrm{H}^{\mathrm{H}}$ phenotype (Fig. 2D, 2E). Interestingly, there was a positive correlation between Ki67 and TP53 mRNA expression in patients with $P T E N^{\mathrm{H}} E S R 1^{\mathrm{H}} P G R^{\mathrm{H}}$ phenotype $(r=0.1644 ; p=0.0216)$ and PTEN ${ }^{\mathrm{L}} E S R 1^{\mathrm{H}} P G R^{\mathrm{H}}$ phenotype $(r=0.1861 ; p=0.0401)$, respectively, but not in PTENLESR1LPGRL, PTEN ${ }^{\mathrm{H}}$ ESR1LPGR ${ }^{\mathrm{L}}$, PTEN $^{\mathrm{L}} E S R 1^{\mathrm{L}} P G R^{\mathrm{H}}$, PTEN $^{\mathrm{L}} E S R 1^{\mathrm{H}}$ $P G R^{\mathrm{L}}, P T E N^{\mathrm{H}} E S R 1^{\mathrm{H}} P G R^{\mathrm{L}}$, and PTEN ${ }^{\mathrm{H}} E S R 1^{\mathrm{L}} P G R^{\mathrm{H}}$ phenotype (Fig. 2F). These results suggest that EC patients with triple-high expression of PTEN, ER and PR showed a lower degree of malignancy and proliferative activity.
A
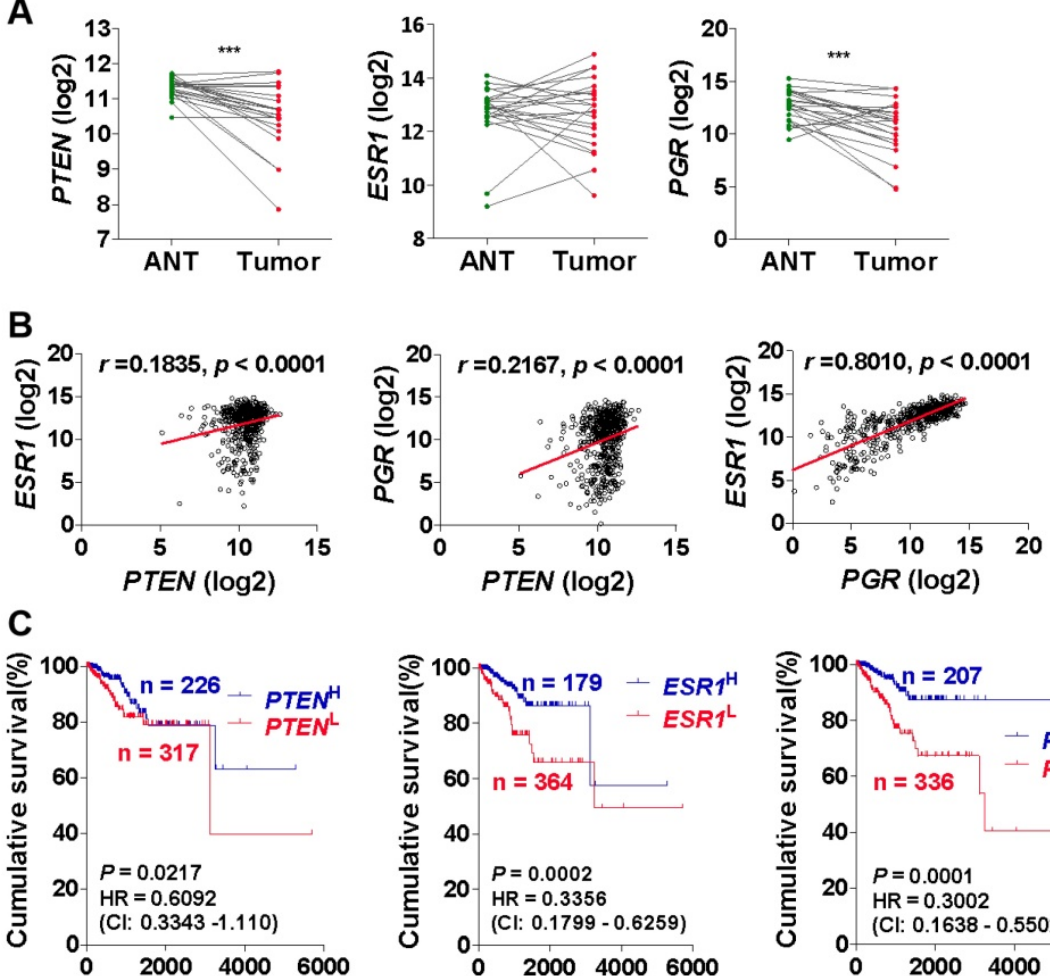

\begin{tabular}{|c|c|}
\hline Group & $\mathbf{N}$ \\
\hline$P T E N^{L} E S R 1^{L} P G R^{L}$ & 22 \\
\hline PTEN'ESR1HPGRH & 122 \\
\hline PTEN ${ }^{H} E S R 1 L P G R^{L}$ & 74 \\
\hline PTENHESR1HPGR ${ }^{H}$ & 195 \\
\hline PTENLESR1LPGRH & 117 \\
\hline PTENLESR1HPGR ${ }^{L}$ & 18 \\
\hline PTENHESR1HPGRL & 30 \\
\hline PTENHESR1LPGR & 19 \\
\hline
\end{tabular}

\section{$\mathbf{F}$}

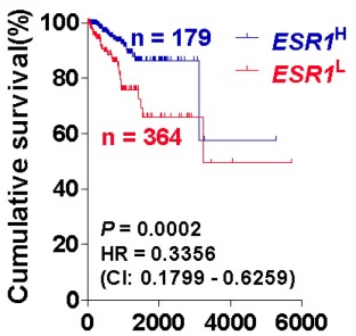

Overall survival time (Days) Overall survival time (Days)

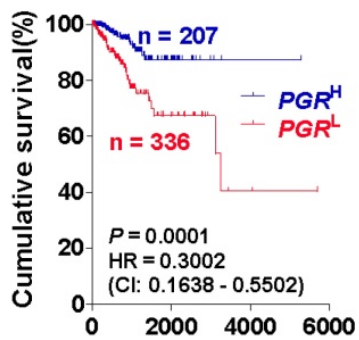

Overall survival time (Days)

\begin{tabular}{|c|c|}
\hline Group & $\mathbf{N}$ \\
\hline PTENLERLPR' & 48 \\
\hline PTENHERLPR & 30 \\
\hline PTENHER ${ }^{H} P R^{H}$ & 20 \\
\hline PTENLER ${ }^{H}$ PR $^{H}$ & 12 \\
\hline PTENHER ${ }^{H} P R^{L}$ & 4 \\
\hline PTEN ${ }^{H} E R^{L P R}{ }^{H}$ & 4 \\
\hline PTENLERHPR' & 2 \\
\hline
\end{tabular}

E
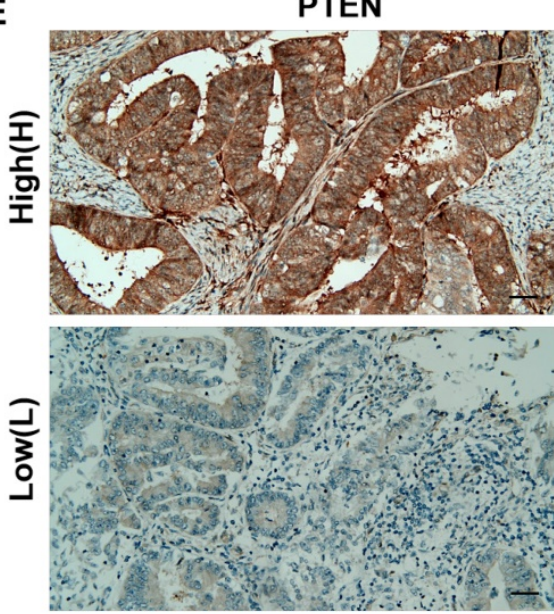

ER

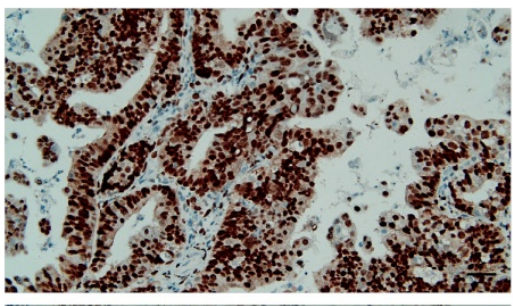

PR

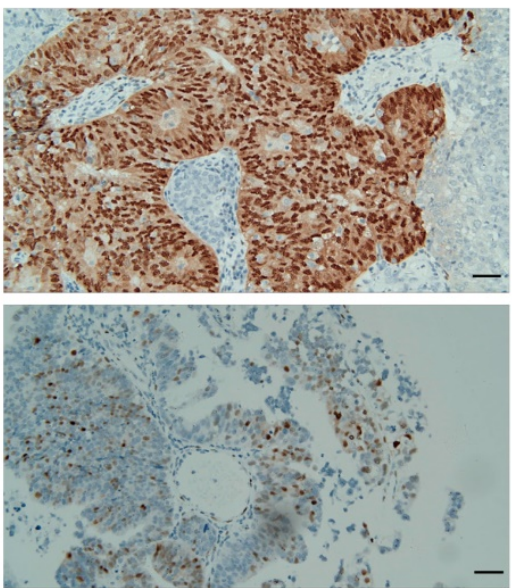

Figure 1. Classification of EC tissues based on PTEN, ER and PR expression. (A) Comparison of PTEN, PGR and ESRI mRNA expression between EC tumor tissues and adjacent normal tissues (ANT) based on TCGA database, respectively. $* * *: p<0.001$. (B) Correlation analysis among PTEN, PGR and ESRI mRNA expression. (C) Kaplan-Meier analysis of overall survival time of EC patients with high PTEN mRNA expression versus low PTEN mRNA expression, high PGR mRNA expression versus low PGR mRNA expression and high ESRI mRNA expression versus low ESRI mRNA expression, respectively, based on TCGA database. (D) EC patients were divided into 8 phenotypes according to high and low PTEN, PGR and ESRI mRNA expression based on TCGA database. (E) Detection of PTEN, ER and PR expression in EC tissues by IHC analysis. (F) EC patients were divided into 7 phenotypes according to high and low PTEN, ER and PR expression based on IHC analysis. H: high, L: low. 
A

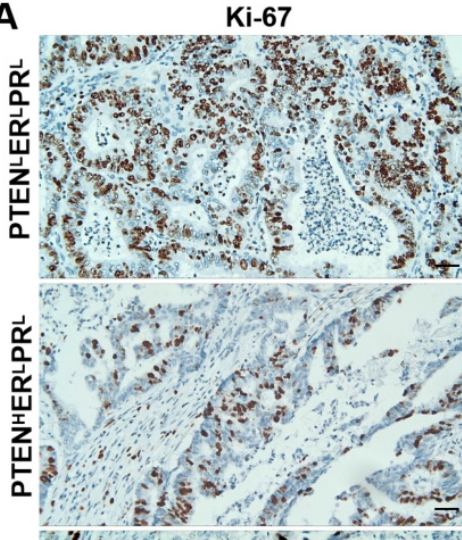

and
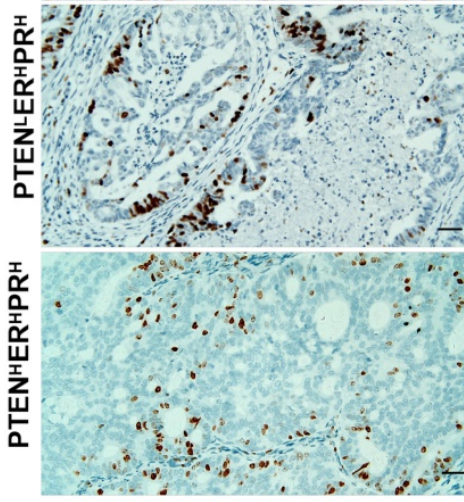

D

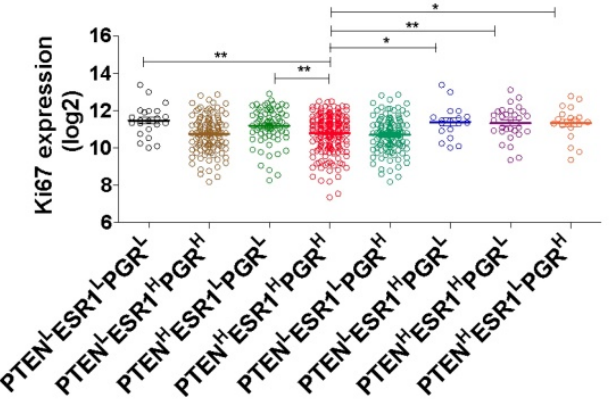

p53

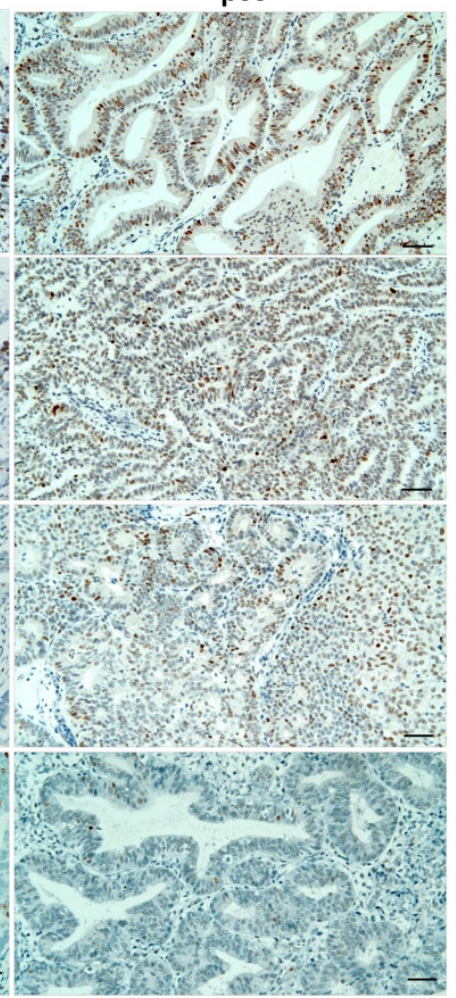

B

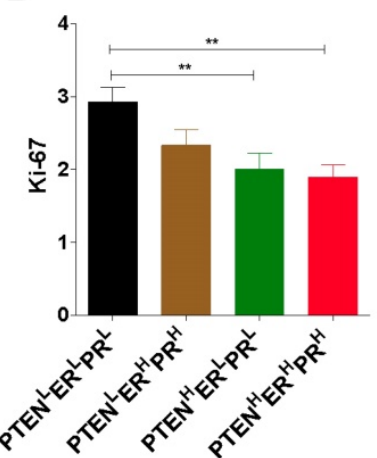

C

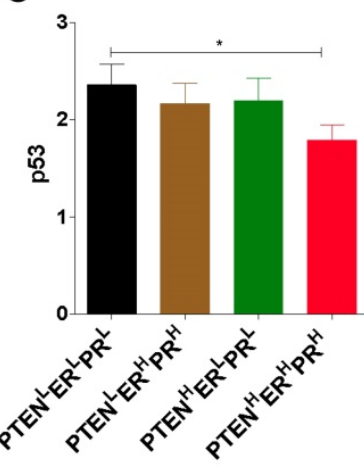

E

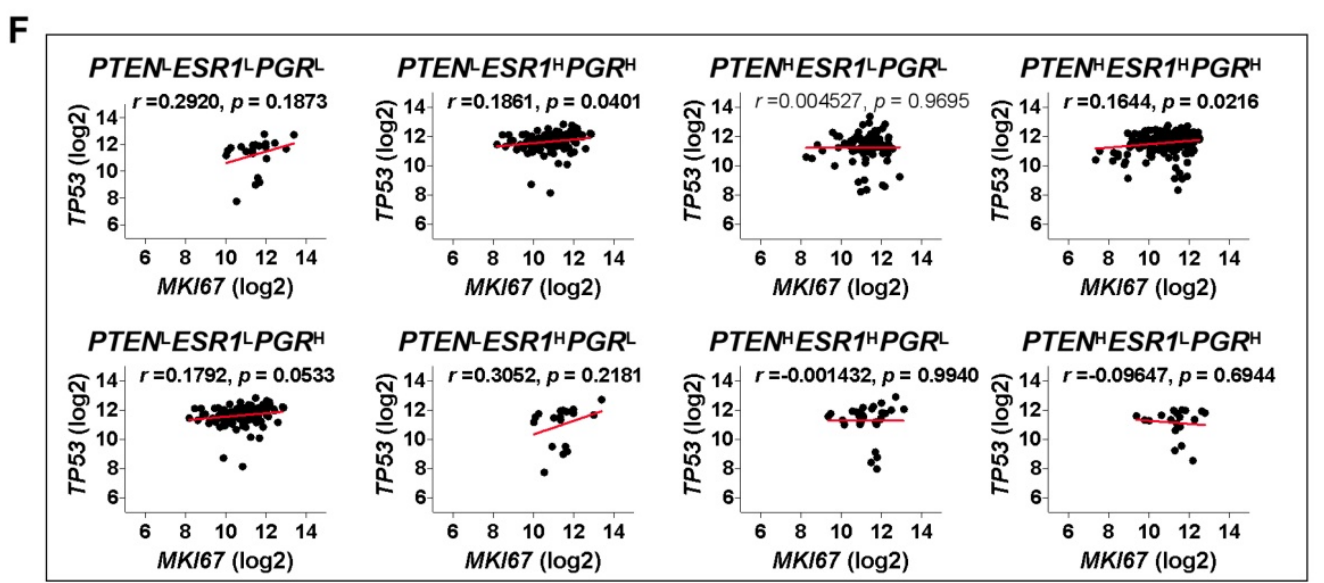

Figure 2. EC patients with triple-H expression of PTEN, ER and PR showed a Ler expression of Ki-67 and p53. (A) Detection of Ki-67 and p53 expression in EC tissues with PTENLERLPRL, PTENHERLPRL, PTENHERHPRH and PTENLERHPRH phenotypes by IHC analysis. (B) Comparison of Ki-67 expression among PTENLERLPRL, PTENHERLPRL, PTENHERHPRH and PTENLERHPRH phenotypes, **. $p<0.01$ (C) Comparison of Ki67 mRNA expression among PTENLESRILPGRL, PTENLESRIHPGRH, PTENHESRILPGRL, PTENHESRIHPGRH, PTENLESRILPGRH, PTENLESRIHPGRL, PTENHESRIHPGRL, and PTENHESRILPGRH phenotyPes based on TCGA database. *: $p<0.05$; **: $p<0.01$. (D) Comparison of 553 expression among PTENLERLPRL, PTENHERLPRL, PTENHERHPRH and PTENLERHPRH phenotypes. **: $p<0.01$. (E) Comparison of TP53 mRNA expression among PTENLESRILPGRL, PTENLESRIHPGRH, PTENHESRILPGRL, PTENHESRIHPGRH, PTENLESRILPGRH, PTENLESRIHPGRL, PTENHESRIHPGRL, and PTENHESRILPGRH phenotypes based on TCGA database. *: $p<0.05$; **: $p<0.01$. (F) Correlation analysis between Ki67 and TP53 mRNA exPression in PTENLESRILPGRL, PTENLESRIHPGRH, PTENHESRILPGRL, PTENHESRIHPGRH, PTENLESRILPGRH, PTENLESRIHPGRL, PTENHESRIHPGR L, and PTENHESRILPGR PhenotyPes based on TCGA database. H: high, L: low. 
Table 1. The demographic and clinical characteristics for all EC phenotypes

\begin{tabular}{|c|c|c|c|c|c|c|c|}
\hline \multirow[t]{2}{*}{ Group } & \multicolumn{7}{|c|}{ EC phenotypes $[\mathrm{n}(\%)]$} \\
\hline & $\begin{array}{l}\text { PTEN lowER lowPR low } \\
(\mathrm{n}=48)\end{array}$ & $\begin{array}{l}\text { PTENhighER lowPR low } \\
(\mathrm{n}=30)\end{array}$ & $\begin{array}{l}\text { PTENhighER highPR high } \\
(\mathrm{n}=20)\end{array}$ & $\begin{array}{l}\text { PTENlowER highPR high } \\
(\mathrm{n}=12)\end{array}$ & $\begin{array}{l}\text { PTENhighER highPR low } \\
(\mathrm{n}=4)\end{array}$ & $\begin{array}{l}\text { PTENhighER low PR high } \\
(\mathrm{n}=4)\end{array}$ & $\begin{array}{l}\text { PTEN lowER highPR low } \\
(\mathrm{n}=2)\end{array}$ \\
\hline \multicolumn{8}{|c|}{ Histological grade } \\
\hline G1 & $10(20.83)$ & $18(60.00)$ & $12(60.00)$ & $5(41.67)$ & $1(25.00)$ & $1(25.00)$ & $1(50.00)$ \\
\hline G2 & $20(41.67)$ & $6(20.00)$ & $5(25.00)$ & $5(41.67)$ & $1(25.00)$ & $1(25.00)$ & $1(50.00)$ \\
\hline G3 & $18(37.50)$ & $6(20.00)$ & $3(15.00)$ & $2(16.67)$ & $2(50.00)$ & $2(50.00)$ & $0(-)$ \\
\hline \multicolumn{8}{|c|}{ FIGO clinical stage } \\
\hline I & $12(25.00)$ & $20(66.67)$ & $9(45.00)$ & $5(41.67)$ & $1(25.00)$ & $2(50.00)$ & $0(-)$ \\
\hline II & $18(37.50)$ & $4(13.33)$ & $7(35.00)$ & $3(25.00)$ & $1(25.00)$ & $1(25.00)$ & $1(50.00)$ \\
\hline III & $13(27.08)$ & $4(13.33)$ & $3(15.00)$ & $3(25.00)$ & $1(25.00)$ & $1(25.00)$ & $1(50.00)$ \\
\hline IV & $5(10.42)$ & $2(6.67)$ & $1(5.00)$ & $1(8.33)$ & $1(25.00)$ & $0(-)$ & $0(-)$ \\
\hline \multicolumn{8}{|c|}{ Myometrial tumor invasion } \\
\hline Yes & $22(45.83)$ & $15(50.00)$ & $8(40.00)$ & $7(58.33)$ & $1(25.00)$ & $2(50.00)$ & $0(-)$ \\
\hline No & $26(54.17)$ & $15(50.00)$ & $12(60.00)$ & $5(41.67)$ & $3(75.00)$ & $2(50.00)$ & $2(50.00)$ \\
\hline \multicolumn{8}{|c|}{ Age (years) } \\
\hline$>45 y$ & $30(62.50)$ & $17(56.67)$ & $12(60.00)$ & $7(58.33)$ & $2(50.00)$ & $3(75.00)$ & $1(50.00)$ \\
\hline$\leq 45 y$ & $18(37.50)$ & $13(43.33)$ & $8(40.00)$ & $5(41.67)$ & $2(50.00)$ & $1(25.00)$ & $1(50.00)$ \\
\hline
\end{tabular}

\section{EC patients with triple-high expression of PTEN, ER and PR showed a lower expression of CA 125}

Carcinoma-associated antigens are considered to be useful markers for the detection of recurrent disease in EC patients [37]. Herein, we detected CA125 and CEA expression in EC tissues by IHC analysis (Fig. 3A). The results show that EC patients with PTEN ${ }^{H} \mathrm{RR}^{\mathrm{H} P \mathrm{R}^{\mathrm{H}}}$ phenotype had low expression of CA125 (Fig. 3B) and EC patients with PTEN ${ }^{\mathrm{H}} E S R 1^{\mathrm{H}} P G R^{\mathrm{H}}$ phenotype had low expression of MUC16 (encoding CA125) mRNA (Fig. 3C). Notably, there was no difference in the expression of CEA among patients with PTENLER ${ }^{L P R}{ }^{L}$, PTENHERLPRL, ${ }^{P T E N}{ }^{H} E^{H}{ }^{H} R^{\mathrm{H}}$, and PTENLER ${ }^{\mathrm{H} P R^{\mathrm{H}}}$ phenotypes (Fig. $3 \mathrm{D})$. However, the mRNA expression of CEACAM5 (encoding CEA) was down-regulated in patients with PTEN ${ }^{\mathrm{H}} E S R 1^{\mathrm{H}} P G R^{\mathrm{H}}$ and PTEN ${ }^{\mathrm{H}} E S R 1^{\mathrm{L}} P G R^{\mathrm{L}}$ phenotype compared to patients with PTENLESR1LPGR ${ }^{\mathrm{H}}$ phenotype based on TCGA-UCEC datasets (Fig. 3E). Furthermore, we observed MUC16 mRNA expression was positively related to CEACAM5 mRNA expression in EC patients with $P T E N^{\mathrm{H}} E S R 1^{\mathrm{H}} P G R^{\mathrm{H}}$ $(r=0.3694 ; p<0.0001)$, PTENLESR1H ${ }^{\mathrm{H}}$ PGR ${ }^{\mathrm{H}}(r=0.3817 ; p$ $<0.0001)$ and PTEN ${ }^{\mathrm{L}} E S R 1^{\mathrm{L}} \mathrm{LGR} \mathrm{H}^{\mathrm{H}}(r=0.3948 ; p<$ 0.0001 ) phenotypes, respectively (Fig. 3F). These studies suggest that there are differences in CEA and CA125 expression in EC patients with different phenotypes. In particular, EC patients with triple-high expression of PTEN, ER and PR showed low expression of CA125, this was positively correlated with CEA.

\section{Triple-high expression of PTEN, ER and PR may predict favorable prognosis in EC patients}

Subsequently, we analyzed the relationship between patients with different EC phenotypes and prognosis, and found that EC patients with
PTEN ${ }^{H}$ ER $^{\mathrm{H}} \mathrm{PR}^{\mathrm{H}}$ phenotype showed a favorable overall survival (OS) time, compared to patients with PTENLER $^{L}{ }^{L}{ }^{L}$ and PTEN ${ }^{H}{ }^{2}{ }^{L}{ }^{L} R^{L}$ phenotype (Fig. 4A). Similarly, EC patients with PTENHESR1 ${ }^{H} P G R^{\mathrm{H}}$, PTENLESR1HPGR $\mathrm{H}$, and PTENHESR1LPGR ${ }^{\mathrm{H}}$ phenotypes showed a favorable OS time than patients with PTEN ${ }^{\mathrm{L}} E S R 1^{\mathrm{L}} P G R^{\mathrm{L}}$, and PTEN ${ }^{\mathrm{H}} E S R 1^{\mathrm{L}} P G R^{\mathrm{L}}$ phenotype (Fig. 4B). These studies suggest that triple-high expression of PTEN, ER and PR may predict favorable prognosis in EC patients.

\section{Discussion}

EC is a type of female reproductive malignant tumor, the incidence of which is generally $20 \sim 30 \%$. Exploring effective molecular prognosis targets for EC patients has become a hot topic in current research. In 1991, Raju KS, et al. found that post medroxyprogesterone acetate (MPA) dehydrogenase enzyme levels predicted survival more accurately than pre MPA receptor status of the tumors in EC patients [38]. Then, some researchers found lack of bcl-2 [39, 40], PTEN [41], TIMP-2 [42], ER and PR [43], decrease of hemoglobin [44], EpCAM [45], up-regulation of serum CA125 [46], serum TIMP-1 [47], ulex europeus agglutinin-I (UEA-I) [48], HIF-1a [49], aurora B [50], MMP-7 [51], homeobox (HOX) transcript antisense intergenic RNA (HOTAIR) [52], CHRM3 [53], AAA+ (ATPases associated with various cellular activities) nuclear coregulator cancer-associated (ANCCA) [54], glycogen synthase kinase-3 $\beta$ (GSK-3 $\beta$ ) [55] can serve as a poor prognostic marker for EC patients. Herein, we also found that decrease of PTEN, PGR and ESR1 mRNA expression were associated with poor prognosis. However, it was not accurate during follow-up. Especially, we found that some EC patients with decrease of PTEN, but up-regulation of ER or PR, and some EC patients with decrease of ER or PR, but up-regulation of PTEN also have a poor prognosis (data not shown). 
A

A
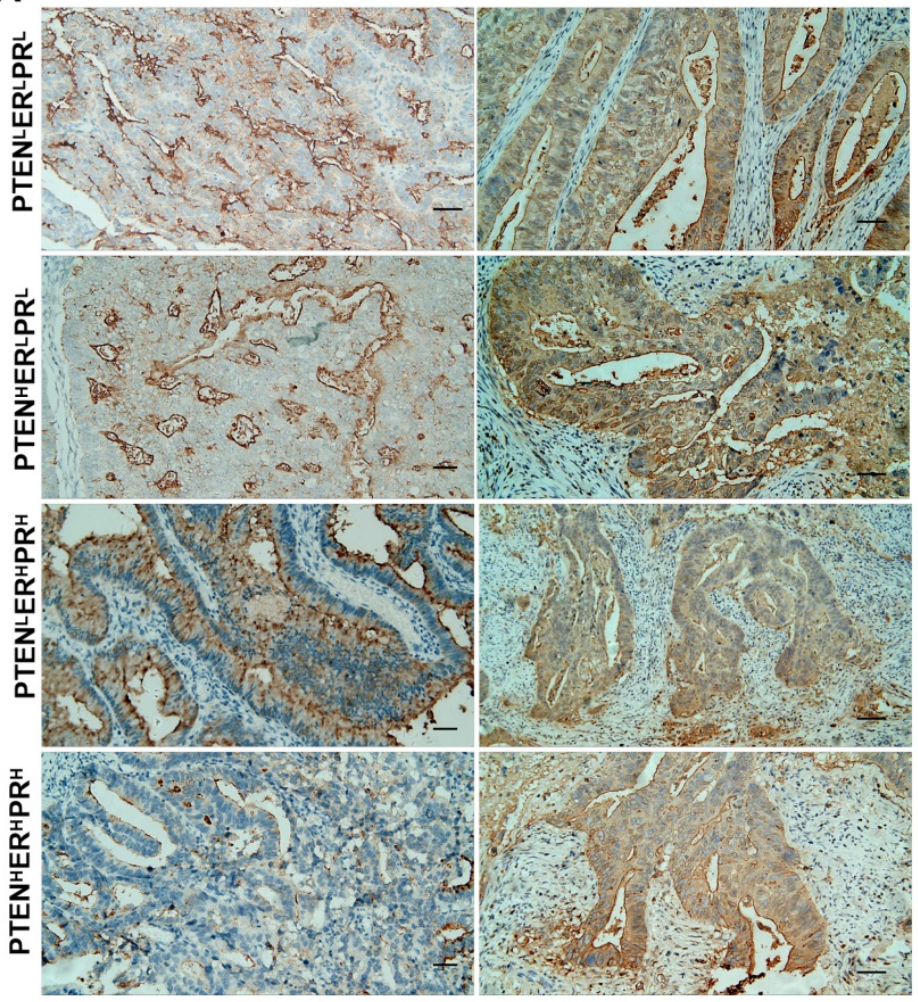

D

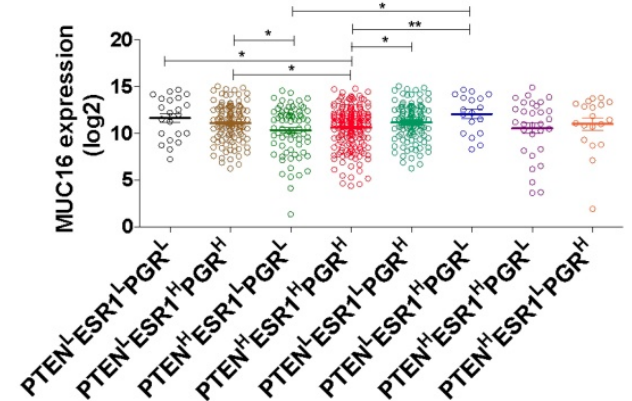

B

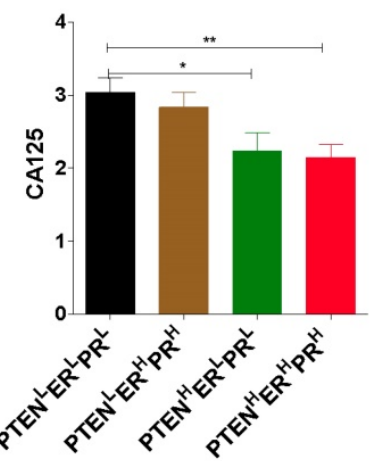

C

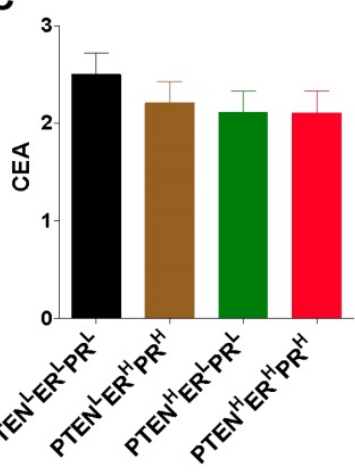

F

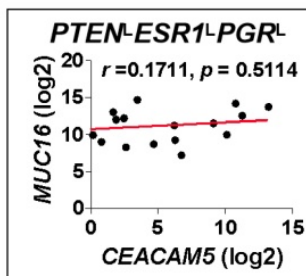

PTEN'ESR1'PGR ${ }^{H}$

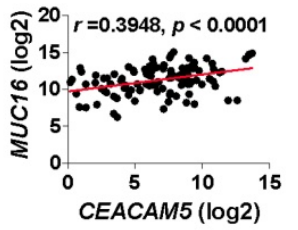

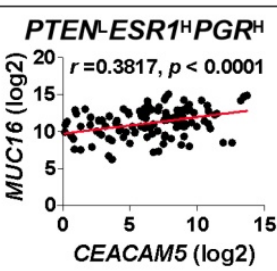

PTEN'ESR1 ${ }^{\text {HPGR }}{ }^{\text {L }}$

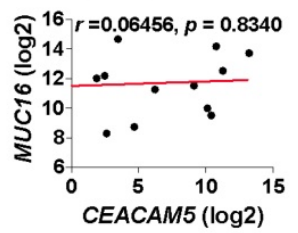

E

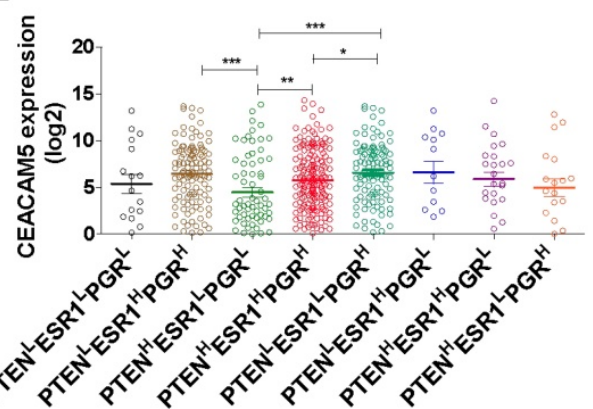

Figure 3. EC patients with triple-H expression of PTEN, ER and PR showed a Ler expression of CA125. (A) Detection of CA125 and CEA expression in EC tissues with PTENLERLPRL, PTENHERLPRL, PTENHERHPRH and PTENLERHPRH phenotypes by IHC analysis. (B) Comparison of CA125 expression among PTENLERLPRL, PTENHERLPRL, PTENHERHPRH and PTENLERHPRH phenotypes. **: $p<0.01$. (C) Comparison of MUCI6 mRNA expression among PTENLESRILPGR', PTENLESRIHPGRH,

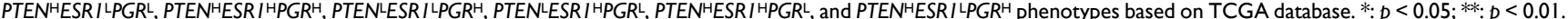

(D) Comparison of CEA expression among PTENLERLPRL, PTENHERLPRL, PTENHERHPRH and PTENLERHPRH phenotypes. **: $p<0.01$. (E) Comparison of CEACAM5 mRNA expression among PTENLESRILPGRL, PTENLESRIHPGR ${ }^{H}$, PTENHESRILPGRL, PTENHESRIHPGRH, PTENLESRILPGRH, PTENLESRIHPGRL, PTENHESRIHPGRL, and PTENHESRILPGRH phenotypes based on TCGA database. *: $p<0.05$; **: $p<0.01$. (F) Correlation analysis between MUCI 6 and CEACAM5 mRNA exPression in PTENLESRILPGRL, PTENLESRIHPGRH, PTENHESRILPGRL, PTENHESRIHPGRH, PTENLESRILPGRH, PTENLESRIHPGRL, PTENHESRIHPGRL, and PTENHESRILPGRH phenotypes based on TCGA database. H: high, L: low. 

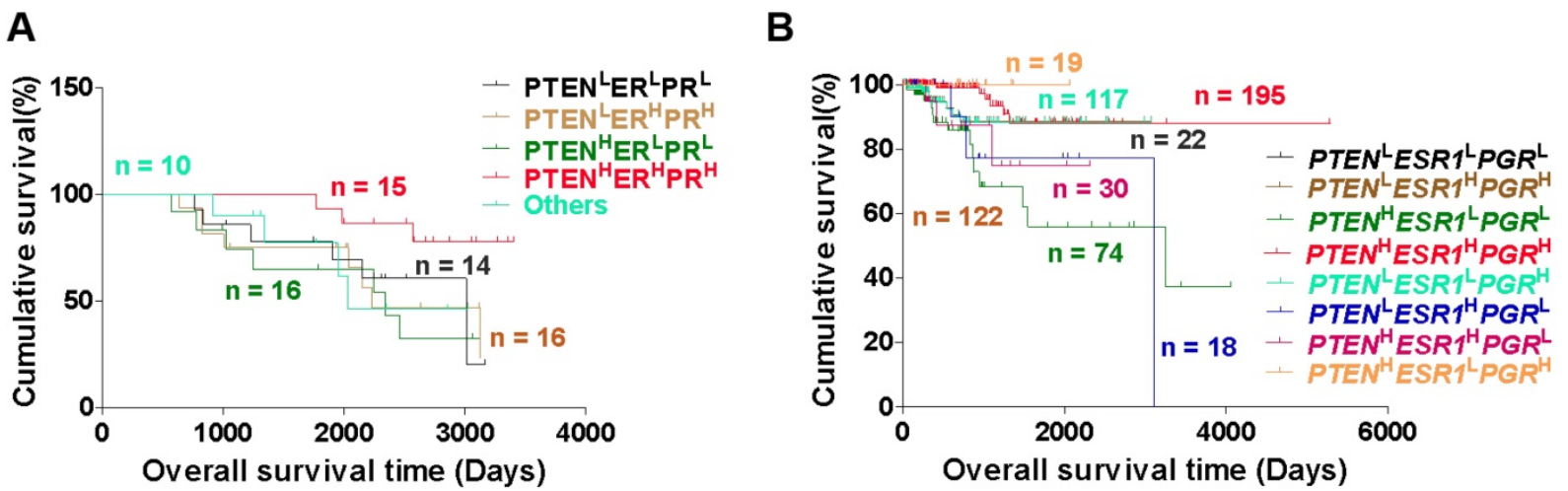

Figure 4. Triple-H expression of PTEN, ER and PR may predict favorable prognosis in EC patients. (A) Kaplan-Meier analysis of overall survival time of EC patients with PTENLERLPRL, PTENHERLPRL, PTENHERHPRH and PTENLERHPRH phenotypes. (B) Kaplan-Meier analysis of overall survival time of EC patients with

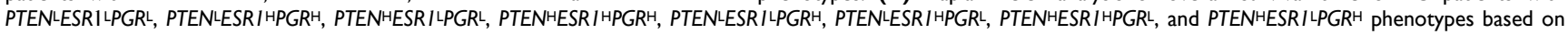
TCGA database. H: high, L: low.

These characteristics can also be observed when analyzing PTEN, PGR and ESR1 mRNA expression using the TCGA database. Indeed, we found that EC patients can be divided into different phenotypes based on PTEN, ER and PR expression. EC patients were divided into PTENLESR1LPGRL, PTENLESR1 ${ }^{\mathrm{H}}$ $P G R^{\mathrm{H}}, \quad P T E N^{\mathrm{H}} E S R 1^{\mathrm{L}} P G R^{\mathrm{L}}, \quad P T E N^{\mathrm{H}} E S R 1^{\mathrm{H}} P G R^{\mathrm{H}}$, PTENLESR1LPGR ${ }^{\mathrm{H}}, \quad$ PTEN ${ }^{\mathrm{L}} E S R 1^{\mathrm{H}} P G R^{\mathrm{L}}, \quad P T E N^{\mathrm{H}}$ ESR $1^{\mathrm{H}} P G R^{\mathrm{L}}$, and $P T E N^{\mathrm{H}} E S R 1^{\mathrm{L}} P G R^{\mathrm{H}}$ phenotypes basing on TCGA-UCEC datasets. Similarly, EC tissues can be classified to PTENLERLPRL, PTEN ${ }^{L} E R^{L} P R L$, PTEN ${ }^{H}{ }^{H}{ }^{H} P R^{H}$, PTENLER ${ }^{H} P R^{\mathrm{H}}$, PTEN ${ }^{H}{ }^{2}{ }^{H} P R$, PTEN ${ }^{H} E R^{L} P R \mathrm{H}$, and PTENLER ${ }^{H}{ }^{2}{ }^{L}$ phenotypes basing on IHC analysis.

PTEN is a tumor suppressor gene with double phosphatase activity discovered in 1997. It is a homologous gene of phosphatase and tensin which often associated with the deletion of chromosome allele 10q site [23]. PTEN plays an important role in the inhibition of tumorigenesis by regulating PTEN/PI3K/AKT, PTEN/FAK/P130cas, PTEN/ ERK, p53/MDM2, and FRAP/mTOR signaling pathways. These signal pathways through its lipid phosphatase and protein phosphatase activity, induce apoptosis, block cell cycle, to inhibit tumor cell invasion and metastasis and tumor angiogenesis [13, 14]. At present, abnormal expression of PTEN in EC patients has been confirmed by most researchers. In the previous study, we also found that the loss rate of PTEN in EC patients was 58.8\% (60/102), and the loss expression of PTEN was closely related to the grade of histological in EC patients, suggesting that the absent expression of PTEN plays an important role on EC occurrence and development [56, 57]. Additionally, the expression of ER and PR may be related to the increase of malignancy, the increase of invasion and the transformation of non-hormone-dependent tumors in EC patients [10-16]. Therefore, the detection of ER and PR may have important value for EC patients' prognosis and treatment. In particular, it is of great significance to detect ER, PR as endocrine therapy marker in EC patients. To reveal the prognosis of EC patients with different phenotypes based on PTEN, ER and PR expression, 120 cases formalin-fixed paraffin-embedded EC tissues and 543 cases UCEC patients from TCGA-UCEC datasets were analyzed. Results showed that EC patients with triple-high expression of PTEN, ER and PR showed a lower expression of Ki-67, p53 and CA125. Furthermore, triple-high expression of PTEN, ER and PR may predict favorable prognosis in EC patients.

Fiorillo, et al. found that the ER-a mutation Y537S, which associated with the over-expression of a number of protein markers of poor clinical outcome (COL6A3, ERBB2, STAT3, AFP, TFF1, CDK4 and CD44) can confer tamoxifen-resistance via enhanced mitochondrial metabolism, glycolysis and Rho-GDI/PTEN signaling [58]. Moreover, PTEN insufficiency stimulated $\mathrm{ER}^{+}$breast cancer cell growth [59]. Additionally, PI3K/PTEN/AKT pathway was crucial for many aspects of cell growth and survival, feedback regulation on PTEN/AKT pathway by the ER stress kinase PERK mediated by interaction with the Vault complex[60]. ER-activating ability of breast cancer stromal fibroblasts was regulated independently of alteration of PTEN [61]. Herein, basing on IHC analysis, EC tissues can be classified to PTENLERLPRL, PTEN ${ }^{H}{ }^{L} R^{L}{ }^{L}{ }^{L}, \quad$ PTEN ${ }^{H}{ }^{2} R^{H}{ }^{2} R^{H}$,

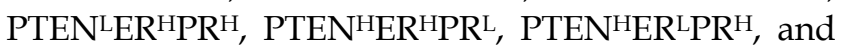
PTENLER ${ }^{H} P R^{L}$ phenotypes. And, patients with PTENLER ${ }^{L} R^{L}$ showed high malignancy, while patients with PTENHER ${ }^{H} P R^{\mathrm{H}}$ showed low malignancy. Therefore, combined detection of PTEN, ER, PR may help identify a small subset of EC with more aggressive behavior and may aid in risk stratification.

In summary, our findings indicate that EC patients with triple-high expression of PTEN, ER and PR showed a lower degree of malignancy and 
proliferative activity and predicted a favorable prognosis. However, the physiological and pathological features of EC patients with different phenotypes basing on PTEN, ER and PR expression still require further investigation.

\section{Abbreviations}

PTEN: phosphatase and tensin homolog; ER: estrogen receptor; PR: progesterone receptor; EC: Endometrial carcinoma; UCEC: uterine corpus endometrial carcinoma; TCGA: the cancer genome atlas; BMI: Body Mass Index; OS: Overall survival; IHC: Immunohistochemistry; GSK-3 $\beta$ : glycogen synthase kinase-3 $\beta$; ANT: adjacent normal tissues.

\section{Acknowledgements}

This study was supported by grants from the Medical Science Foundation of Guangdong Province (A2018123), the Science and Technology Project of Dongguan (201750715005451), and Science and Technology Project of Guangdong Province (2014A020212298).

\section{Author Contributions}

LY and ZJ conceived and designed the experiments. $\mathrm{LY}, \mathrm{LB}$ and $\mathrm{CC}$ performed the experiments. CS, ZX and $\mathrm{YH}$ analyzed the data. LY, ZJ and $\mathrm{ZK}$ participated in writing manuscript. The final version of the manuscript has been reviewed and approved for publication by every author.

\section{Ethics Committee Approval and Patient Consent}

All procedures performed in studies involving human participants were in accordance with the ethical standards of the Research Ethics Committee of Dongguan Fifth People's Hospital (China).

\section{Competing Interests}

The authors have declared that no competing interest exists.

\section{References}

1. Ren $Y$, Huang $X$, Shan B, et al. Adjuvant concurrent chemoradiation followed by chemotherapy for high-risk endometrial cancer. Gynecologic oncology. 2016; 140: 58-63

2. Beavis AL, Smith AJ, Fader AN. Lifestyle changes and the risk of developing endometrial and ovarian cancers: opportunities for prevention and management. International journal of women's health. 2016; 8: 151-67.

3. Morice P, Leary A, Creutzberg C, et al. Endometrial cancer. Lancet. 2016; 387: 1094-108.

4. Amant F, Moerman P, Neven P, et al. Endometrial cancer. Lancet. 2005; 366: 491-505.

5. Kandoth $\mathrm{C}$, Schultz $\mathrm{N}$, Cherniack $\mathrm{AD}$,et al. Integrated genomic characterization of endometrial carcinoma. Nature. 2013; 497: 67-73.

6. Murali R, Soslow RA, Weigelt B. Classification of endometrial carcinoma: more than two types. The Lancet. Oncology. 2014; 15: e268-78.

7. Lewin SN, Wright JD. Comparative Performance of the 2009 International Federation of Gynecology and Obstetrics' Staging System for Uterine Corpus Cancer. Obstetrics and gynecology. 2011; 117: 1226.

8. Söderlin E. Factors affecting prognosis of endometrial carcinoma. Acta obstetricia et gynecologica Scandinavica. Supplement. 1974; 38: 1-37.
9. Beckner ME, Mori T, Silverberg SG. Endometrial carcinoma: nontumor factors in prognosis. International journal of gynecological pathology : official journal of the International Society of Gynecological Pathologists. 1985; 4: 131-45.

10. Tian W, Teng F, Zhao J, et al. Estrogen and insulin synergistically promote type 1 endometrial cancer progression. Cancer biology \& therapy. 2017; 18: 1000-10.

11. Abd ENM, El-Gelany S. Differential Expression Patterns of PTEN in Cyclic, Hyperplastic and Malignant Endometrium: Its Relation with ER, PR and Clinicopathological Parameters. Journal of the Egyptian National Cancer Institute. 2009; 21: 323-31.

12. Kappes H, Goemann C, Bamberger AM, et al. PTEN expression in breast and endometrial cancer: correlations with steroid hormone receptor status. Pathobiology : journal of immunopathology, molecular and cellular biology. 2001; 69: 136-42.

13. Pieczyńska B, Wojtylak S, Zawrocki A, et al. Analysis of PTEN, estrogen receptor $a$ and progesterone receptor expression in endometrial hyperplasia using tissue microarray. Polish journal of pathology : official journal of the Polish Society of Pathologists. 2011; 62: 133-8.

14. Peevey JF, Seagle BL, Maniar KP, et al. Association of body mass index with $\mathrm{ER}, \mathrm{PR}$ and 14-3-3o expression in tumor and stroma of type I and type II endometrial carcinoma. Oncotarget. 2017; 8: 42548-59.

15. NCM V, van der Wurff AAM, JMA P, et al. Tissue microarray is suitable for scientific biomarkers studies in endometrial cancer. Virchows Archiv : an international journal of pathology. 2018; 472: 407-13.

16. Stoenescu VE, Niculescu M, Novac L,et al. Immunohistochemical reaction of the glandular epithelium in endometrial hyperplasia compared to endometrial carcinoma. Romanian journal of morphology and embryology $=$ Revue roumaine de morphologie et embryologie. 2017; 58: 791-800.

17. Kininis M, Kraus WL. A global view of transcriptional regulation by nuclear receptors: gene expression, factor localization, and DNA sequence analysis. Nuclear receptor signaling. 2008; 6: e005.

18. Ethier JL, Desautels DN, Amir E, et al. Is hormonal therapy effective in advanced endometrial cancer? A systematic review and meta-analysis. Gynecologic oncology. 2017; 147: 158-66.

19. Zhang $Y$, Zhao D, Gong C, et al. Prognostic role of hormone receptors in endometrial cancer: a systematic review and meta-analysis. World journal of surgical oncology. 2015; 13: 208.

20. Huvila J, Talve L, Carpén O, et al. Progesterone receptor negativity is an independent risk factor for relapse in patients with early stage endometrioid endometrial adenocarcinoma. Gynecologic oncology. 2013; 130: 463-9.

21. Estrella JS, Broaddus RR, Mathews A, et al. Progesterone receptor and PTEN expression predict survival in patients with low- and intermediate-grade pancreatic neuroendocrine tumors. Archives of pathology \& laboratory medicine. 2014; 138: 1027-36.

22. Skrzypczak M, Lattrich C, Häring J, et al. Expression of SCUBE2 gene declines in high grade endometrial cancer and associates with expression of steroid hormone receptors and tumor suppressor PTEN. Gynecological endocrinology : the official journal of the International Society of Gynecological Endocrinology. 2013; 29: 1031-5.

23. Bilbao C, Rodríguez G, Ramírez R, et al. The relationship between microsatellite instability and PTEN gene mutations in endometrial cancer. International journal of cancer. 2006; 119: 563-70.

24. Huang $\mathrm{P}$, Zhang $\mathrm{H}$, Zhang $\mathrm{XF}$, et al. Evaluation of Intraductal Ultrasonography, Endoscopic Brush Cytology and K-ras, P53 Gene Mutation in the Early Diagnosis of Malignant Bile Duct Stricture. Chinese medical journal. 2015; 128: 1887-92.

25. Werner HM, Trovik J, Marcickiewicz J, et al. Revision of FIGO surgical staging in 2009 for endometrial cancer validates to improve risk stratification. Gynecologic oncology. 2012; 125: 103-8.

26. Lu Z, Chen J. Introduction of WHO classification of tumours of female reproductive organs, fourth edition. Zhonghua bing li xue za $\mathrm{zhi}=$ Chinese journal of pathology. 2014; 43: 649-50.

27. Liang Y, Chen Q, Du W, et al. Epstein-Barr Virus-Induced Gene 3 (EBI3) Blocking Leads to Induce Antitumor Cytotoxic T Lymphocyte Response and Suppress Tumor Growth in Colorectal Cancer by Bidirectional Reciprocal-Regulation STAT3 Signaling Pathway. Mediators of inflammation. 2016; 2016: 3214105 .

28. Liu XJ, Feng JS, Xiang WY, et al. Clinicopathological features of an ascending colon mixed adenoneuroendocrine carcinoma with clinical serosal invasion. International journal of clinical and experimental pathology. 2014; 7: 6395-8.

29. Zeng JC, Zhang Z, Li TY, et al. Assessing the role of IL-35 in colorectal cancer progression and prognosis. International journal of clinical and experimental pathology. 2013; 6: 1806-16

30. Zhang X, Zhang L, Lin B, et al. Phospholipid Phosphatase 4 promotes proliferation and tumorigenesis, and activates Ca2+-permeable Cationic Channel in lung carcinoma cells. Molecular cancer. 2017; 16: 147.

31. Ren D, Lin B, Zhang X, et al. Maintenance of cancer stemness by miR-196b-5p contributes to chemoresistance of colorectal cancer cells via activating STAT3 signaling pathway. Oncotarget. 2017; 8: 49807-23.

32. Li J, Yen C, Liaw D, et al. PTEN, a putative protein tyrosine phosphatase gene mutated in human brain, breast, and prostate cancer. Science. 1997; 275: 1943-7.

33. Tashiro $\mathrm{H}$, Blazes MS, $\mathrm{Wu} \mathrm{R}$, et al. Mutations in PTEN are frequent in endometrial carcinoma but rare in other common gynecological malignancies. Cancer research. 1997; 57: 3935-40. 
34. Guigon CI, Zhao L, Willingham MC, et al. PTEN deficiency accelerates tumour progression in a mouse model of thyroid cancer. Oncogene. 2009; 28: 509-17.

35. Armstrong CW, Maxwell PJ, Ong CW, et al. PTEN deficiency promotes macrophage infiltration and hypersensitivity of prostate cancer to IAP antagonist/radiation combination therapy. Oncotarget. 2016; 7: 7885-98.

36. Petrocelli $T$, Slingerland JM. PTEN deficiency: a role in mammary carcinogenesis. Breast cancer research : BCR. 2001; 3: 356-60.

37. Bruns F, Micke O, Halek G, et al. Carcinoembryonic antigen (CEA)--a useful marker for the detection of recurrent disease in endometrial carcinoma patients. Anticancer research. 2003; 23: 1103-6.

38. Raju KS, King RJ, Taylor RW. Biochemical changes in endometrial carcinoma and their correlation to clinical outcome and prognosis. European journal of gynaecological oncology. 1991; 12: 331-9.

39. Sakuragi N, Ohkouchi T, Hareyama H, et al. Bcl-2 expression and prognosis of patients with endometrial carcinoma. International journal of cancer. 1998; 79: 153-8.

40. Geisler JP, Geisler HE, Wiemann MC, et al. Lack of bcl-2 persistence: an independent prognostic indicator of poor prognosis in endometrial carcinoma. Gynecologic oncology. 1998; 71: 305-7.

41. Kanamori Y, Kigawa J, Itamochi H, et al. PTEN expression is associated with prognosis for patients with advanced endometrial carcinoma undergoing postoperative chemotherapy. International journal of cancer. 2002; 100: 686-9.

42. Honkavuori-Toivola M, Talvensaari-Mattila A, Soini $\mathrm{Y}$, et al. Immunoreactivity for TIMP-2 is associated with a favorable prognosis in endometrial carcinoma. Tumour biology : the journal of the International Society for Oncodevelopmental Biology and Medicine. 2012; 33: 935-41.

43. Li M, Wang Z, Zhao L, et al. Predictors of recurrence and prognosis in patients with stage I and II endometrial carcinoma. Zhonghua fu chan ke za zhi. 2014; 49: 455-9.

44. Tamussino KF, Gücer F, Reich O, et al. Pretreatment hemoglobin, platelet count, and prognosis in endometrial carcinoma. International journal of gynecological cancer : official journal of the International Gynecological Cancer Society. 2001; 11: 236-40.

45. Wen KC, Sung PL, Chou YT, et al. The role of EpCAM in tumor progression and the clinical prognosis of endometrial carcinoma. Gynecologic oncology. 2018; 148: 383-92.

46. Xie Z, Zhang J, Tan A. Relationship between serum CA125 level and prognosis in the patients with uterine endometrial carcinoma. Zhonghua yi xue za zhi. 2001; 81: 1456-7.

47. Honkavuori M, Talvensaari-Mattila A, Puistola U, et al. High serum TIMP-1 is associated with adverse prognosis in endometrial carcinoma. Anticancer research. 2008; 28: 2715-9.

48. Ookuma Y, Hachisuga T, Iwasaka T, et al. Assessment of lectin binding for prognosis in endometrial carcinoma. Pathology. 1994; 26: 225-9.

49. Sivridis E, Giatromanolaki A, Gatter $\mathrm{KC}$, et al. Association of hypoxia-inducible factors 1alpha and 2alpha with activated angiogenic pathways and prognosis in patients with endometrial carcinoma. Cancer. 2002; 95: 1055-63.

50. Kurai M, Shiozawa T, Shih HC, et al. Expression of Aurora kinases A and B in normal, hyperplastic, and malignant human endometrium: Aurora B as a predictor for poor prognosis in endometrial carcinoma. Human pathology. 2005; 36: 1281-8.

51. Misugi F, Sumi T, Okamoto E, et al. Expression of matrix metalloproteinases and tissue inhibitors of metalloproteinase in uterine endometrial carcinoma and a correlation between expression of matrix metalloproteinase-7 and prognosis. International journal of molecular medicine. 2005; 16: 541-6.

52. He X, Bao W, Li X, et al. The long non-coding RNA HOTAIR is upregulated in endometrial carcinoma and correlates with poor prognosis. International journal of molecular medicine. 2014; 33: 325-32.

53. Wang Y, Li J, Wen S, et al. CHRM3 is a novel prognostic factor of poor prognosis in patients with endometrial carcinoma. American journal of translational research $2015 \cdot 7 \cdot 902-11$.

54. Shang P, Meng F, Liu Y, et al. Overexpression of ANCCA/ATAD2 in endometrial carcinoma and its correlation with tumor progression and poor prognosis. Tumour biology : the journal of the International Society for Oncodevelopmental Biology and Medicine. 2015; 36: 4479-85.

55. Chen S, Sun KX, Liu BL, et al. The role of glycogen synthase kinase-3 $\beta$ (GSK-3 $\beta$ ) in endometrial carcinoma: A carcinogenesis, progression, prognosis, and target therapy marker. Oncotarget. 2016; 7: 27538-51.

56. Liang YF, Zeng JC, Kang DP, et al. The expression and clinical significance of PTEN,P27 and BAG-1 in endometrial carcinoma. Chongqing Med. 2014; 43: 785-7.

57. Liang YF, Ruan JB, Kang DP, et al. Expression of estrogen receptor and progesterone receptor on PTEN-deficient endometrial carcinoma. J Clin Exp Patho. 2015; 31

58. Fiorillo M, Sanchez-Alvarez R, Sotgia F, et al. The ER-alpha mutation Y537S confers Tamoxifen-resistance via enhanced mitochondrial metabolism, glycolysis and Rho-GDI/PTEN signaling: Implicating TIGAR in somatic resistance to endocrine therapy. Aging. 2018; 10: 4000-23.

59. Chiang KC, Chen HY, Hsu SY, et al. PTEN insufficiency modulates ER+ breast cancer cell cycle progression and increases cell growth in vitro and in vivo. Drug design, development and therapy. 2015; 9: 4631-8.

60. Zhang W, Neo SP, Gunaratne J, et al. Feedback regulation on PTEN/AKT pathway by the ER stress kinase PERK mediated by interaction with the Vault complex. Cellular signalling. 2015; 27: 436-42.
61. Suda T, Oba H, Takei H, et al. ER-activating ability of breast cancer stromal fibroblasts is regulated independently of alteration of TP53 and PTEN tumor suppressor genes. Biochemical and biophysical research communications. 2012; 428: 259-63. 